\title{
Three-dimensional dynamics of relativistic flows in pair plasmas with force-free magnetic configuration
}

\author{
Laboratory for Plasma Astrophysics, Faculty of Engineering \\ Akira Matsuo, Jun-ichi Sakai and Michihiro Hosokawa \\ m032240@ems.toyama-u.ac.jp
}

\begin{abstract}
We investigate the dynamics of the relativistic flow in pair plasmas with force-free magnetic configuration, using a three-dimensional fully relativistic electromagnetic particle-in-cell (PIC) code. We observed that three-dimensional Alfvén waves with magnetic helical structures can be excited through complicated three-dimensional tearing instability triggered from the streaming instability. During these dynamical processes the pair plasma can be heated through the magnetic field dissipation and also the high-energy particles are generated.
\end{abstract}

keyword : force-free、relativistic flow, pair plasma, Alfvén wave

\section{Introduction}

It is assumed in astrophysical plasmas that the relativistic jet or the relativistic plasma flow associated with the gamma-ray bursts [1] may interact with the surrounding stable force-free configuration of the magnetic field. Our motivation is to study what happens under this situation and energy conversion between the kinetic energy of plasma flow and the magnetic field energy. Haruki and Sakai [2] investigated the dynamics of the relativistic flow in force-free magnetic field in pair plasmas, using a two-dimensional fully relativistic and electromagnetic PIC code. They found that during the early stage of the interaction there occurs the streaming instability, that induces the electromagnetic perturbations associated with generation of quasi-static magnetic field.

We investigate the dynamics of the relativistic flow in pair plasmas, using a three-dimensional fully relativistic electromagnetic PIC code. This study is an extension of the work by Haruki and Sakai [2] that was done in a two-dimensional force-free magnetic configuration. We found here that the threedimensional configuration could result in completely different dynamics, except for the initial phase where the streaming instability develops.

\section{Simulation model}

This simulation code used here is threedimensional fully relativistic electromagnetic particle-in-cell code. Our simulation model is drawn in Figure 1.

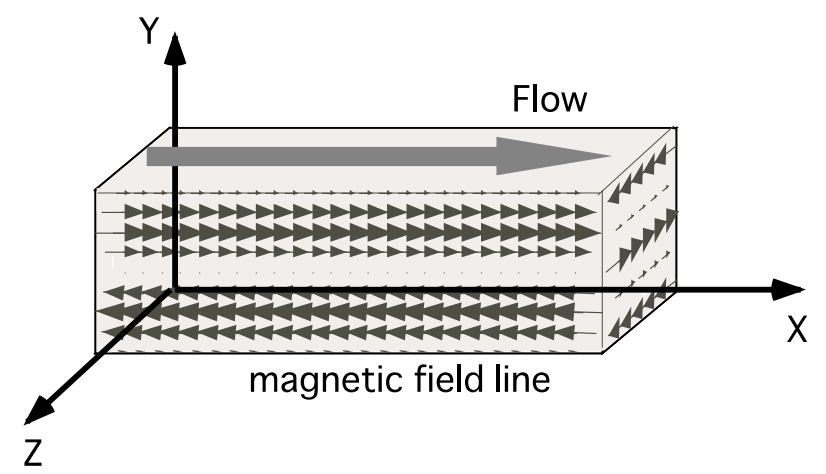

Figure 1: Simulation model

The system size is $L_{x}=300 \Delta$ and $L_{y}=80 \Delta$ and $L_{z}=115 \Delta$, where $\Delta(=1)$ is the grid size. Periodic boundary conditions are imposed on particles, background plasma keeping a force-free magnetic configuration and relativistic streaming plasmas of uniform density with a shifted Maxwellian of velocity of $0.8 \mathrm{c}$ 
in the x-direction. In Figure 1, the large cursor shows the flows direction. There are $1.38 \times 10^{7}$ electronpositron pairs filling the entire domain uniformly corresponding to the background plasma, keeping the domain charge neutral. Hence the average particle number density of the background is about 4 per cell, while the average particle number density of the relativistic streaming plasma is about 1 per cell. We consider a simple force-free configuration with current sheets, whose magnetic fields are given by

$$
\begin{gathered}
B_{x}(y)=B_{0} \sin \left(\frac{y-40}{L}\right), \\
B_{y}(y)=0, \\
B_{z}(y)=B_{0} \cos \left(\frac{y-40}{L}\right),
\end{gathered}
$$

where $\mathrm{L}\left(=\mathrm{E}_{y} / 2 \pi\right)$ is the width of the current sheets. Figure 1, the small cursors show the magnetic fields direction. The line of the center of the current sheets in the $x-y$ plane is located at $y=0,40,80$, and in the $\mathrm{y}-\mathrm{z}$ plane is located at $y=20,60$. To generate the above magnetic fields, we impose shifted Maxwell distributions with a drift velocity of $v_{d}=$ $0.131 c=1.31 v_{t e}$ in $\mathrm{x}$ - and z-directions for both electron and positron velocity distributions. This configuration is stable against current-driven Buneman instability. The initial state is characterized by complete charge neutrality $\left(E_{0}=0\right)$ everywhere in the domain. Other parameters are as follows: the mass ratio between an electron and a positron, $m_{e} / m_{p}=1$; the simulation time step $\omega_{c e} / \omega_{p e}=0.133$, the ratio of the background electron thermal velocity parallel to the magnetic field $B_{x}$ to the light velocity $V_{t e} / c=0.1$; the Debye length $\lambda_{D e}=0.89 \Delta$; the skin depth $c / \omega_{p e}=10.0 \Delta$; the plasma beta value $\beta=0.57$; the ratio of the temperature of electrons $T_{e}$ to the temperature of positrons $T_{p}, T_{e} / T_{p}=1$. The gyroradius for both thermal electrons and positrons is $\rho_{e}=\rho_{p}=7.5 \Delta$. The thermal velocity of the relativistic flow plasma is $0.1 V_{t e}$. To confirm the accuracy of our simulations, we checked the energy conservation during the whole simulation time that is about $0.97 \%$.

\section{Simulation results}

\subsection{Time history of energies}
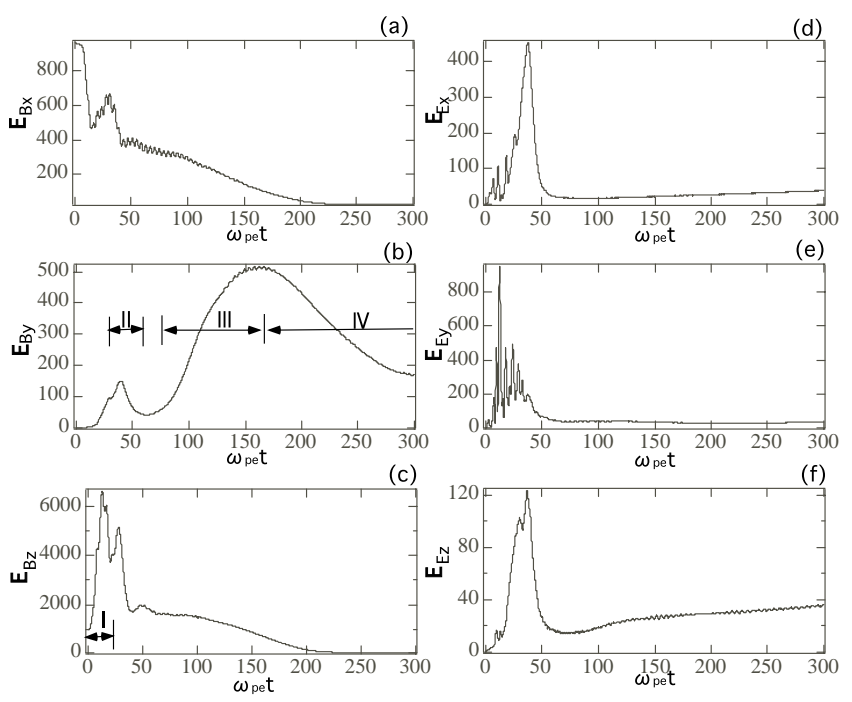

Figure 2: The time development of the electric and magnetic field energies (a) $E_{B x}=1 /\left(2 \mu_{0}\right) \iiint \mathrm{B}_{x}^{2} d x d y d z$, (b) $E_{B y}=1 /\left(2 \mu_{0}\right) \iiint \mathrm{B}_{y}^{2} d x d y d z$,

(c) $E_{B z}=1 /\left(2 \mu_{0}\right) \iiint \mathrm{B}_{y}^{2} d x d y d z$, (d) $E_{E x}=1 / 2 \epsilon_{0} \iiint \mathrm{E}_{x}^{2} d x d y d z$, (e) $E_{E y}=1 / 2 \epsilon_{0} \iiint \mathrm{E}_{y}^{2} d x d y d z$, (f) $E_{E z}=1 / 2 \epsilon_{0} \iiint \mathrm{E}_{z}^{2} d x d y d z$.

In this section we present our simulation results. We found that the dynamical interaction between the force-free magnetic configuration and the relativistic plasma flows develops sequentially through four different physical processes: (I) The phase of streaming instability, (II) The phase of magnetic reconnection triggered by the first streaming instability, (III) The phase of Alfvén wave excitation through the reconnection process and (VI) The phase of dissipation of the Alfvén waves through the magnetic reconnection. During these dynamical phases the pair plasma can be heated through the magnetic field dissipation and also the high-energy particles are generated. In Fig. 2 we present the time history of the magnetic and electric field energies in the system. The above four physical processes during which time period they occur are indicated in Figs.2(c) and (b). 

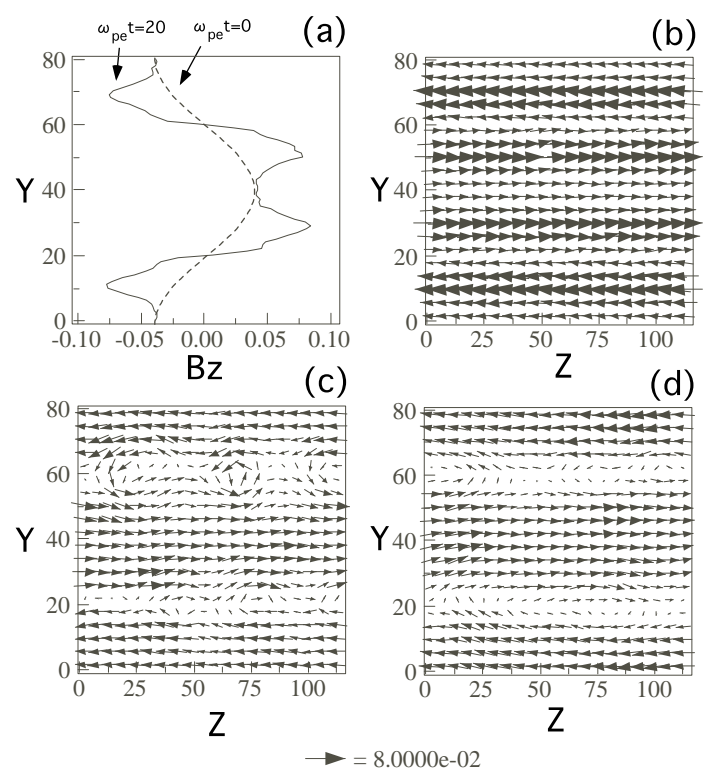

Figure 3: (a) The line plot of $B_{z}$ at $\omega_{p e} \mathrm{t}=0$, and the vector plot of $B_{y}-B_{z}$ in the $\mathrm{y}-\mathrm{z}$ plane cut by $\mathrm{x}=150$ at (b) $\omega_{p e} \mathrm{t}=20$, (c) $\omega_{p e} \mathrm{t}=40,(\mathrm{~d}) \omega_{p e} \mathrm{t}=60$.

\subsection{Four different physical processes}

As seen from Fig. 2(c), in first step, the initial flow energy decreases associated with the generation of magnetic field $B_{z}$ during the streaming instability.

In Fig. 3(a) we show the lineplots of $B_{z}$ at $\omega_{p e} \mathrm{t}=0$ (dashed line) and $\omega_{p e} \mathrm{t}=20$ (solid line) on $\mathrm{x}=150 \Delta$ and $z=57 \Delta$. As seen in this figure, the initial currents are strongly compressed due to the generation of the magnetic field $B_{z}$ during nonlinear stage of the streaming instability. Therefore the collisionless tearing instability can be induced in the thinning current sheets with the thickness of the order of the electron skin depth. As seen in Fig. 3(b)-(d) showing the time development of the magnetic field lines in the $y$ $\mathrm{z}$ plane on $\mathrm{x}=150 \Delta$ at three time steps: (b) $\omega_{p e} \mathrm{t}=20$, (c) $\omega_{p e} \mathrm{t}=40$, and (d) $\omega_{p e} \mathrm{t}=60$, the magnetic islands are formed during development of the tearing instability. In Fig. 4 we present the time development of the magnetic field lines in the $\mathrm{x}-\mathrm{y}$ plane on $\mathrm{z}=57 \Delta$ at four time steps:(a) $\omega_{p e} \mathrm{t}=0,(\mathrm{~b}) \omega_{p e} \mathrm{t}=20,(\mathrm{c}) \omega_{p e} \mathrm{t}=40$, and $(\mathrm{d}) \omega_{p e} \mathrm{t}=60$. As seen in Fig.4(c), the tearing instability is very weakly excited in the current sheets in the $x-y$ plane, because the thinning of the current sheets in the $x-y$ plane is very weak due to the streaming instability.

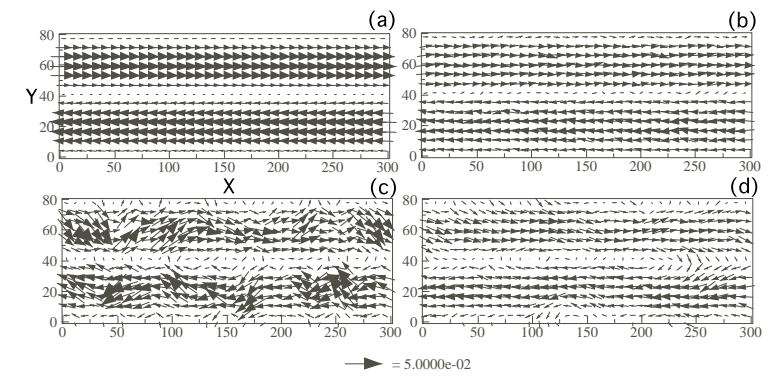

Figure 4: The vector plot of $B_{x}-B_{y}$ in the x-y plane cut by $\mathrm{z}=58 \Delta$ at (a) $\omega_{p e} \mathrm{t}=0,(\mathrm{~b}) \omega_{p e} \mathrm{t}=20,(\mathrm{c}) \omega_{p e} \mathrm{t}=40,(\mathrm{~d}) \omega_{p e} \mathrm{t}=60$.

(a)

(b)
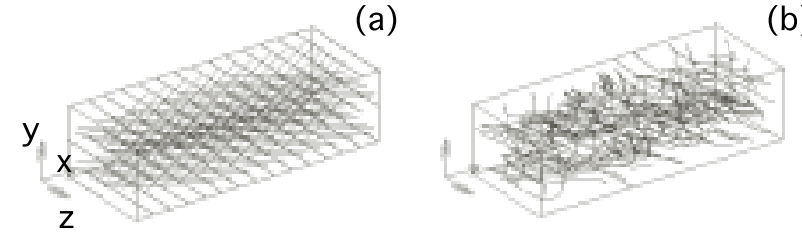

(c)

(d)
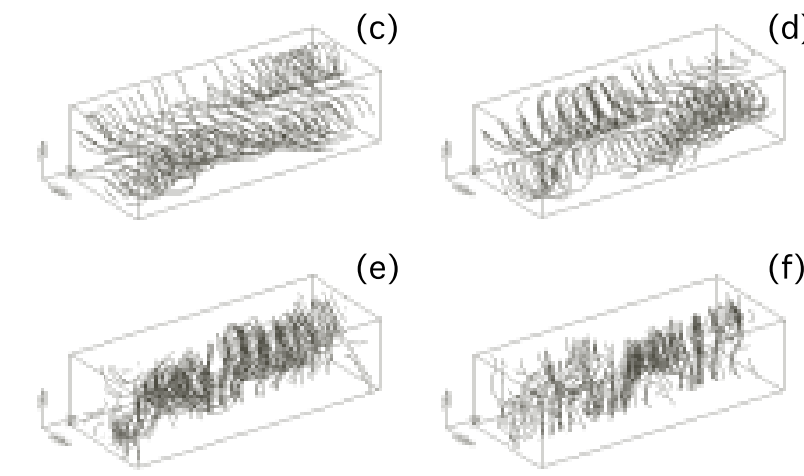

(e)

(f)

Figure 5: The time development of the magnetic field line at (a) $\omega_{p e} \mathrm{t}=0,(\mathrm{~b}) \omega_{p e} \mathrm{t}=50,(\mathrm{c}) \omega_{p e} \mathrm{t}=100, \quad(\mathrm{~d}) \omega_{p e} \mathrm{t}=150$, (e) $\omega_{p e} \mathrm{t}=200,(\mathrm{f}) \omega_{p e} \mathrm{t}=250$.

In Fig. 5 we present the time development of the magnetic field lines from the initial state $(\mathrm{a}) \omega_{p e} \mathrm{t}=0$ to the final time of the simulation, (f) $\omega_{p e} \mathrm{t}=250$. As seen in Fig.5 (b) that corresponds to the phase (II) in the previous section, the three-dimensional structure of the magnetic field lines is very complicated. However, as seen in Fig.5 (c), the disordered magnetic field line structure develops to very coherent structure with two helical magnetic field structures during the phase (III). As seen from Figs. 6(d)-(f), the black region with positive value of $B_{y}$ propagate in the positive $\mathrm{x}$-direction in time. The propagation speed estimated from these figures is about the Alfvén wave speed. This implies that the excited coherent mag- 

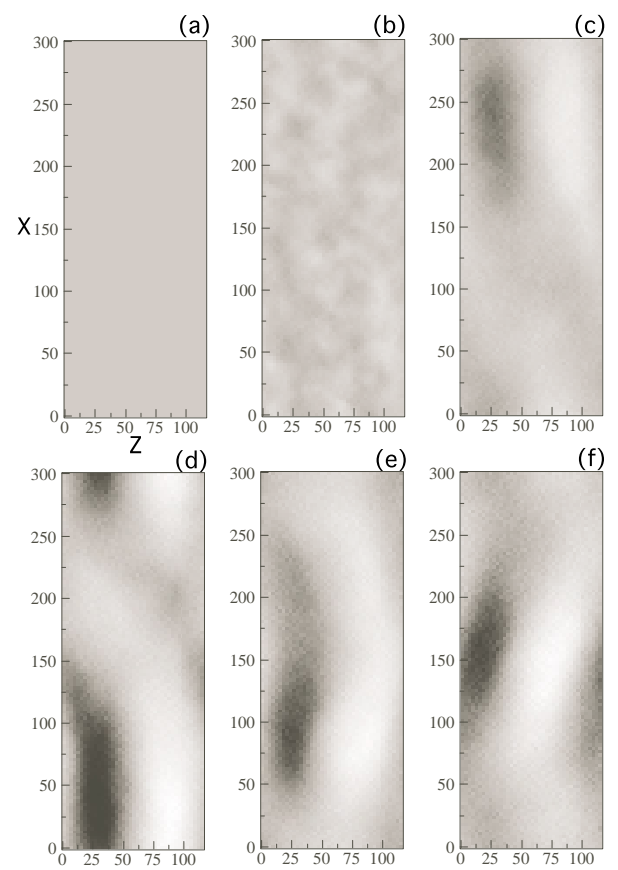

(e)

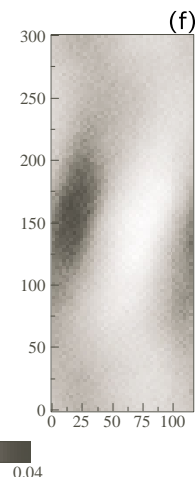

Figure 6: The time development of $B_{y}$ in the z-x plane cut by $\mathrm{y}=40$ at (a) $\omega_{p e} \mathrm{t}=0,(\mathrm{~b}) \omega_{p e} \mathrm{t}=50,(\mathrm{c}) \omega_{p e} \mathrm{t}=100$, (d) $\omega_{p e} \mathrm{t}=150$, (e) $\omega_{p e} \mathrm{t}=200,(\mathrm{f}) \omega_{p e} \mathrm{t}=250$.

netic helical structures are the Alfvén waves propagating to the $\mathrm{x}$-direction. The dispersion relation of $B_{y}$ along the $\mathrm{x}$-axis is close to the theoretical dispersion relation of Alfén waves.

We investigate the last phase where the Alfvén waves excited in the third phase dissipate their energy through two step magnetic reconnection processes. The first magnetic reconnection occurs during the merging process of two helical structures, as seen in Figs.5(d) and 5(e). While the second magnetic reconnection occurs after the state in Fig.5(e), because due to periodic boundary condition the same helical structures exist in the y-direction. Positron velocity distribution functions are the same as the electron ones. We find that the initial kinetic flow energy can be eventually dissipated through the above three different physical processes and be converted to the heating of pair plasmas as well as the high-energy particle production. About $23 \%$ of the initial flow energy was converted to the plasma heating. About $87.3 \%$ of the initial magnetic field energy was dissipated through magnetic reconnection processes.

\section{Conclusions}

We investigated the dynamics of the relativistic flow in force-free magnetic field in pair plasmas, using a three-dimensional fully relativistic electromagnetic particle-in-cell code. We found here that the threedimensional configuration resulted in completely different dynamics compared with the 2-D case, except for the initial phase where the streaming instability develops. The dynamical interaction between the force-free magnetic configuration and the relativistic plasma flows develops sequentially through four different physical processes: (I) The phase of streaming instability, (II) The phase of magnetic reconnection triggered by the first streaming instability, (III) The phase of Alfvén wave excitation through the reconnection process and (VI) The phase of dissipation of the Alfvén waves through the magnetic reconnection. We observed that three-dimensional Alfvén waves with magnetic helical structures can be excited through complicated three-dimensional tearing instability triggered from the streaming instability. During these dynamical processes the pair plasma can be heated through the magnetic field dissipation and also the high-energy particles are generated. The interaction process between the force-free collision-less plasmas and the relativistic plasma flows may play an important role for the effective magnetic field energy dissipation, formation of filament structures and high-energy particle production in astrophysical plasmas.

\section{References}

[1] T. Piran, Phys. Rep. 314, 575 (1999).

[2] T. Haruki and J. I. Sakai, Phys. Plasmas, 8, 1538,2001

[3] O. Buneman, in Computer Space Plasma Physics, Simulation Techniques and Software, edited by H. Matsumoto and Y. Omura (Terra Scientific, Tokyo, 1993), P67 